Published in final edited form as:

Vaccine. 2018 April 12; 36(16): 2220-2225. doi:10.1016/j.vaccine.2017.11.045.

\title{
Effect of influenza vaccine on Tumor necrosis factor-like weak inducer of apoptosis (TWEAK) in older adults
}

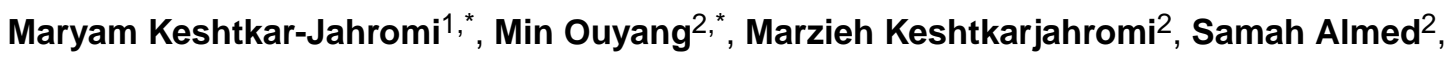 \\ Huifen Li $^{2}$, Jeremy D. Walston ${ }^{2}$, Rebeca Rios ${ }^{3}$, and Sean X. Leng ${ }^{2}$ \\ ${ }^{1}$ Medicine/ Infectious Diseases, Johns Hopkins University School of Medicine, Baltimore, MD \\ ${ }^{2}$ Medicine/ Geriatrics, Johns Hopkins University School of Medicine, Baltimore, MD \\ ${ }^{3}$ Biostatistics, Epidemiology, and Database (BEAD) Core; Department of Medicine, Johns \\ Hopkins University School of Medicine, Baltimore, MD
}

\begin{abstract}
Influenza immunization is recommended for older adults annually, and has been reported to have cardiovascular protective effects. TNF-related weak inducer of apoptosis (TWEAK), an inflammatory mediator implicated in the development of cardiovascular diseases, could be a mechanism for such effect. The objective of this study was to evaluate the effect of influenza vaccine on TWEAK levels. In this study individuals older than 70 were recruited during 20072008 season and immunized with the standard trivalent influenza vaccine. Frailty was evaluated using a validated set of criteria. Sera were collected immediately before and during the $4^{\text {th }}$ week
\end{abstract}

Addresses: Maryam Keshtkar-Jahromi, MD, MPH, Corresponding Author, Johns Hopkins University School of Medicine, 5200 Eastern AV, Medicine/ Infectious Diseases, Baltimore, MD, Phone: 410-550-1291 (office), 443-802-1291 (cell), Fax: 410-550-1169, maryam.keshtkar@jhmi.edu.

*M K-J and M O contributed to this work equally.

Min Ouyang, MD, Johns Hopkins University School of Medicine, 4940 eastern avenue, Medicine/Geriatrics, Baltimore, MD 21224, Phone: 410-550-4778, Mindy.gao@163.com

Marzieh Keshtkarjahromi, MD, Johns Hopkins University School of Medicine, 4940 EASTERN AVENUE, Medicine/Geriatrics, Baltimore, MD, Phone: 4437421645, mkeshtkar-jahromi@ som.umaryland.edu

Samah Almed, MS, Johns Hopkins University School of Medicine, 4940 eastern avenue, Medicine/Geriatrics, Baltimore, MD,

Phone: 420-550-4778, sahmed8@ neomed.edu

Huifen Li, PhD, Johns Hopkins University School of Medicine, 4940 eastern avenue, Medicine/Geriatrics, Baltimore, MD 21224, Phone: 410-550-4778, hli55@jhmi.edu

Jeremy D. Walston, MD, Johns Hopkins University School of Medicine, 4940 eastern avenue, Medicine/Geriatrics, Baltimore, MD, Phone: 410-550-0286, JWALSTON@jhmi.edu

Rebeca Rios, PhD, Johns Hopkins University School of Medicine, 5200 Eastern Ave, MFL Center Tower, 2nd floor, Biostatistics, Epidemiology, and Database (BEAD) Core; Department of Medicine, Baltimore, MD 21224, Phone: 410-550-0524, rrios2@ jhmi.edu Sean X. Leng, MD, PhD, Johns Hopkins University School of Medicine, Johns Hopkins Asthma and Allergy Center, Room 1A.38A, 5501 Hopkins Bayview Circle, Medicine/Geriatrics, Baltimore, MD 21224, Phone: 410-550-2494, sleng1@jhmi.edu

Publisher's Disclaimer: This is a PDF file of an unedited manuscript that has been accepted for publication. As a service to our customers we are providing this early version of the manuscript. The manuscript will undergo copyediting, typesetting, and review of the resulting proof before it is published in its final citable form. Please note that during the production process errors may be discovered which could affect the content, and all legal disclaimers that apply to the journal pertain.

Authors' contribution

M.K-J., O.M., M.K., S.A., H.L., and S.X.L participated in the study design, acquisition and analyzing data, and writing the article. R.R. was the primary statistician who performed descriptive and inferential analyses. All authors participated in critically reviewing the article.

Conflict of interest

Dr. Sean Leng receives high dose influenza vaccine supply from Sanofi-Pasteur for his current study. Other authors declare no conflicts of interest.

The result of this study was presented as a poster at IDWeek 2016, New Orleans, LA. 
after vaccination. Pre- and post-vaccination levels of TWEAK, soluble CD163 (sCD163) and strain-specific influenza antibody titers were measured in 69 participants. Multiple regression analyses were employed to examine the effect of influenza vaccine on TWEAK and SCD163, adjusting for age, gender, and hypertension. Post-vaccination TWEAK [mean \pm standard deviation $(\mathrm{SD})=591.7 \pm 290.1 \mathrm{pg} / \mathrm{ml}]$ was significantly lower than pre-vaccination level $(690.6 \pm 330.0 \mathrm{pg} / \mathrm{ml})$ $(\mathrm{p}=0.003)$. No significant difference was observed between pre and post-vaccination sCD163 levels $(\mathrm{p}=0.71)$. Post-vaccination TWEAK levels were significantly higher in men $(\mathrm{p}=0.01)$ and participants with college or higher level of education $(\mathrm{p}=0.044)$. There was no significant difference in post-vaccination TWEAK according to other demographics or pre-existing medical conditions. A 2-fold or greater antibody titer against H1N1 vaccine strain was associated with a more pronounced reduction in TWEAK at the $\mathrm{p}<.10$ level $(\mathrm{p}=0.091)$. A time by frailty interaction term $(p=0.091)$ indicated that the vaccination-induced reduction of TWEAK was greatest among frail individuals. These results of this observational study indicate that the impact of Influenza vaccine on TWEAK, including the role of specific antibody responses of specific vaccine strains and frailty status, warrants further investigation. Such investigation may elucidate whether this effect plays a role in mediating cardiovascular protection of influenza vaccination.

\section{Keywords}

Influenza vaccine; TWEAK; sCD163; frailty; cardiovascular disease

\section{Introduction}

Cardiovascular diseases (CVDs) are the most common cause of death worldwide. In the US, about 610,000 people die from heart disease annually. CVDs are common in older adults and account for $25 \%$ of its all-cause mortality in the US [1,2]. Cardiovascular protective effect of influenza vaccination has been reported in multiple studies [3-10]. A number of recent large systematic reviews have also provided cumulative evidence for such effect [1113]. However, potential molecular and immunological mechanisms underlying such cardiovascular protective effect beyond prevention of influenza infection have yet to be determined.

Tumor necrosis factor (TNF)-related weak inducer of apoptosis (TWEAK) is a TNF superfamily member, a pro-inflammatory molecule primarily produced by leukocytes, the circulating immune cells, with intracellular mRNA and protein expression detected in resting and activated monocytes, dendritic cells (DCs), NK cells, as well as T and B lymphocytes [14]. Fn14, the smallest TNF receptor superfamily member and the only known signaling receptor for TWEAK, is not expressed by circulating immune cells (such as T or B lymphocytes), but mainly by epithelial cells, endothelial cells and other non-hematopoietic cells [15-17]. Fn14 is normally expressed at relatively low levels in healthy tissues and TWEAK triggers its inducible expression [16]. The TWEAK/Fn14 pathway has been recognized as a critical "immune switch" that plays an important role in a number of physiologic and pathophysiological processes including inflammation, apoptosis, and fibrosis [14]. For example, TWEAK/Fn14 pathway activation may play an important role in contributing to CVDs and their severity. Results from a study of patients who were 
hospitalized for CVDs that measured TWEAK levels within 24 hours after hospital admission demonstrated significantly higher TWEAK levels in patients with ST elevation myocardial infarction (STEMI) compared to controls or patients with stable CVDs. TWEAK levels were also shown to be a predictor of adverse short-term outcome after STEMI [18]. In addition to the clinical study cited above, evidence from animal studies suggest that TWEAK/Fn14 pathway is a positive regulator of cardiac hypertrophy [19]. In addition, deletion of the gene encoding Fn14 has been shown to be protective against myocardial fibrosis and heart failure. Genetic deletion of TWEAK and treatment with a TWEAK blocking antibody in atherosclerosis-prone mice has shown reduction in atherosclerosis burden and lesion size in aorta with enhanced plaque stability [20], suggesting the critical role of TWEAK/Fn14 pathway in the development of atherosclerosis and plaque instability. Taken together, TWEAK/Fn14 pathway activation may play an important role in the development of CVDs and, as such, has been proposed as a promising interventional target for CVDs and congestive heart failure [21]. The objective of this study was to evaluate potential effect of influenza vaccination on circulating TWEAK levels in older adults. We hypothesized that influenza vaccination would decrease circulating TWEAK levels. To test this hypothesis, we conducted an analysis comparing TWEAK levels in pre- and postvaccination serum samples collected in a prospective observational study of influenza vaccination among community-dwelling older adults. We also evaluated associations of vaccine-induced strain-specific antibody responses and frailty of older persons with their post-vaccination TWEAK levels.

\section{Material and Methods}

\subsection{Human subjects}

This analysis was conducted in cryopreserved serum samples collected in a prospective observational study of influenza immunization among community-dwelling older adults. Its main finding of the impact of frailty on vaccine-induced strain-specific antibody responses and clinical effectiveness of influenza vaccination was reported elsewhere [22]. Briefly, participants were recruited from the community during 2007-2008 influenza season. The study was advertised via collaborating physicians and community newspaper, flyers at clinics, senior citizen buildings, and retirement places in Baltimore, Maryland. Individuals older than 70 years of age were eligible except those with history of egg allergy or reaction to influenza vaccine or those who had acute viral infection or acute worsening of chronic diseases (chronic obstructive pulmonary disease, etc.) at the time of the study, active rheumatoid arthritis or other inflammatory conditions or cancer, severe dementia (unable to provide informed consent), Parkinson's disease, or recent usage (within past year) of immune suppressant or modulating agents including oral steroids, chemotherapy or radiation therapy. These exclusion criteria were employed to eliminate allergic reaction to influenza vaccine, minimize potential impact of preexisting medical conditions or medication on the immune system including antibody response to influenza vaccine or development of frailty. Participants were screened by trained clinical research coordinators. Demographic data, clinical diagnoses, list of medications, Mini-Mental State Examination (MMSE) score and data on measurements of frailty were recorded by interviewing and testing the participants as well as reviewing their medical records. Frailty status was determined according to Fried 
screening criteria of frailty phenotype [23]. The criteria include slowed motor performance (measured by 15 -ft walking), self-reported exhaustion, weakness (measured by grip strength), unintentional weight loss and low physical activity. Older persons with three or more criteria were defined as frail; those with one or two as prefrail; and those with none as nonfrail. Standardized Folstein MMSE was used as cognitive screening tool [24]. All participants were interviewed individually and signed the written consent. The Johns Hopkins University Institutional Review Board has approved the study protocol.

\subsection{Influenza immunization}

Participants were recruited in early October during influenza season 2007-2008. After initial screening and signing written informed consents, participants were seen at Clinical Research Unit (CRU) of Johns Hopkins Institute of Clinical and Translational Research on Johns Hopkins Bayview Medical Center (JHBMC) campus, or home for pre-vaccination evaluation. After pre-vaccination blood sample was obtained, $0.5 \mathrm{ml}$ of standard influenza trivalent inactivated vaccine (TIV) recommended for 2007-2008 season (Fluarix, GlaxoSmithKline) was administered intramuscularly by a licensed health-care provider. This standard dose TIV contains $15 \mathrm{mcg}$ of hemagglutinin antigen for each of the 3 influenza virus strains, A/Solomon Islands/3/2006 (H1N1), A/Wisconsin/67/2005 (H3N2), and B/ Malaysia/2506/2004. Participants were followed either at JHBMC CRU or home for postvaccination evaluation and blood draw during the $4^{\text {th }}$ week after vaccination. Sera were isolated using centrifugation for 20 minutes and stored at $-80^{\circ} \mathrm{C}$ for subsequent measurement of serum TWEAK, sCD163 levels and influenza vaccine strain-specific antibody titers.

\subsection{Strain-specific antibody titer measurement}

Antibody titers against each strain were measured by hemagglutination inhibition (HI) assay as previously described [22]. All reference antigens and anti-sera were obtained from WHO Collaborating Center for the Surveillance, Epidemiology and Control of Influenza, Centers for Disease Control and Prevention (CDC), and National Center for Immunization and Other Respiratory Diseases, Influenza division (Atlanta, GA). Receptor Destroying Enzyme (RDE) was used to eliminate non-specific inhibitors of hemagglutination in sera specimens (DENKA SEIKEN CO., LTD). Non-specific agglutinins were removed by pre-adsorption with turkey red blood cells (RBC) (Fitzgerald Industries International, Concord, MA). HI antibody titers were measured after reference antigens were titrated against turkey RBC and reference sera. V-shaped 96-well microtiter plates were used to measure HI titers based on standard microtiter technique. Pre and post-vaccination $\mathrm{HI}$ titer against each vaccine antigen was measured at the same time for each participant. HI titers were recorded as the reciprocal of the highest serum dilution that produced complete inhibition of RBC agglutination.

\subsection{Measurement of pre- and post-vaccination serum levels of TWEAK and SCD163}

Pre- and post-vaccination serum levels of TWEAK and SCD163 were measured using commercially available ELISA according to the procedures provided by the manufacturer. TWEAK was measured using human TWEAK DuoSet ELISA with a detectable range of $62.50-4,000 \mathrm{pg} / \mathrm{mL}$ and an inter-assay coefficient of variance (CV) of 3.9\% (R\&D Systems, Minneapolis, MN). sCD163 was measured using human CD163 Quantikine 
ELISA Kit (R\&D Systems, Minneapolis, MN) with a detectable range of $1.6-100 \mathrm{ng} / \mathrm{mL}$ and an inter-assay CV of $4.7 \%$. Measurements were performed in duplicate and repeated if the measures differed by more than $15 \%$ or were out of the measureable range. The average of the 2 values in duplicate was used for analysis.

\subsection{Statistical analysis}

Summary statistics of demographic and clinical characteristics, and mean TWEAK and sCD163 levels across sample characteristics, were compiled for descriptive purposes using one-way ANOVAs. To test whether TWEAK and SCD163 levels significantly changed due to vaccination, we used repeated measures ANOVAs. To test associations between degree of antibody response and TWEAK, we regressed post-vaccination TWEAK levels on antibody responses to influenza vaccine $\mathrm{H} 1 \mathrm{~N} 1, \mathrm{H} 3 \mathrm{~N} 2$, and $\mathrm{B}$ strains in separate linear regressions. Because of low frequency of participants with greater than 2-fold antibody response, 2-fold, 4-fold and 8-fold seroconversion levels were combined, yielding binary variables with 2 levels representing, 1-fold (or non-response) vs. 2-fold or greater antibody response. Fully adjusted multivariable models controlled for pre-vaccination TWEAK, gender, age, and history of hypertension. All predictors were centered at the mean. The association between frailty status and TWEAK was examined using a mixed ANOVA with one within-subjects factor (pre vs. post-vaccination) and one between-subjects factor for frailty status. Due to the pilot nature of the study and limited sample size, we considered a higher probability of type I error to be appropriate and considered significance tests at the $p>.05$ and $p>.10$ to indicate evidence for statistically significant trends. All analyses were conducted using SPSS version 2013 (SPSS Inc. Released 2012. IBM SPSS Statistics for Windows, Version 23.0. Armonk, NY: IBM Corp.).

\section{Results}

\subsection{Characteristics of the study participants}

Of 94 persons initially screened, $78(83 \%)$ met the eligibility criteria and were enrolled into the study. Seven individuals were lost to follow-up due to either moving out of the area for the winter season $(n=4)$, refusal of providing a post-vaccination blood specimen $(n=2)$, or being hospitalized after an accidental fall and subsequent death $(n=1)$. Two participants were excluded for whom TWEAK values were missing. This yielded a final sample size of 69 ( $88 \%$ of the total enrolled) (Figure. 1). Table 1 summarizes major demographic and clinical characteristics of the study population. The mean age was 84.5 years $(\mathrm{SD}=4.6)$ with a range of 72 to 95 ; most participants were white $(91.6 \%)$ and female (77.5\%). There were significant differences in mean post-vaccination TWEAK according to sex $(\mathrm{p}=0.010)$ and education $(\mathrm{p}=0.044)$.

\subsection{Impact of influenza vaccination on TWEAK and SCD163 levels}

Post-vaccination TWEAK levels (mean+ standard deviation $(\mathrm{SD})=591.7 \pm 290.1 \mathrm{pg} / \mathrm{ml}$ ) were significantly lower than pre-vaccination levels $(690.6 \pm 330 \mathrm{pg} / \mathrm{ml})(\mathrm{p}=0.003)$; Postvaccination sCD163 levels (342191.3 $\pm 162032.1 \mathrm{pg} / \mathrm{ml})$ was not significantly different compared to pre-vaccination levels $(352757.65 \pm 166114.1 \mathrm{pg} / \mathrm{ml})(\mathrm{p}=0.71)$. One-way ANOVAs yielded no significant differences in post-vaccination TWEAK levels according to 
age, race, frailty status, mini mental state examination (MMSE) score, and history of hypertension, heart failure, diabetes mellitus, heart attack, stroke, or cancer. Post-vaccination TWEAK levels were significantly higher in men $(p=0.01)$ and participants with college or some graduate work $(\mathrm{p}=0.044)$. Figure 2 illustrates comparison between pre- and postvaccination TWEAK levels.

\subsection{Association between antibody response to influenza vaccine and post-vaccination TWEAK levels}

A multiple regression model predicting post-vaccination TWEAK while holding prevaccination TWEAK constant indicated that antibody response to $\mathrm{H} 1 \mathrm{~N} 1$ influenza vaccine strain was associated with TWEAK $(\mathrm{B}=-106.7, \mathrm{SE}=54.3, \mathrm{p}=.054)$. This association indicates that the adjusted difference in TWEAK for those who had a 2-fold or greater response to H1N1 influenza vaccine strain was $106.7 \mathrm{pg} / \mathrm{ml}$ less than those with a 1 -fold response. Mean TWEAK for a 1-fold response was 751.9, and the mean for a 2-fold or greater response was 645.3. In the final model adjusting for gender, age, and history of hypertension, the effect of strain-specific antibody response to H1N1 was significant at $\mathrm{p}<$. 10. Table 2 lists regression results for the fully adjusted model of post-vaccination TWEAK levels on H1N1 strain-specific antibody response. Similarly adjusted regression models examining the effect of antibody response to $\mathrm{H} 3 \mathrm{~N} 2$ or B influenza vaccine strains individually on post-vaccination TWEAK levels showed no significant associations in either model (H3N2 strain: $\mathrm{B}=17.0, \mathrm{SE}=57.9, \mathrm{p}=.77$ and $\mathrm{B}$ strain: $\mathrm{B}=3.11, \mathrm{SE}=89.5, \mathrm{p}=.97$, respectively).

\subsection{Association between frailty and post-vaccination TWEAK levels}

To test whether the change in pre- to post-vaccination TWEAK levels varied according to frailty level, we tested the interaction term in a mixed ANOVA of the within-subjects factor (pre vs. post) and the between-subjects factor frailty. The interaction term was significant at the $\mathrm{p}>.10$ level, $(\mathrm{F}(1,65)=2.48, \mathrm{p}=.091)$, suggesting a significant trend given the small sample size. Figure 3 shows that the drop in TWEAK levels from pre-vaccination to postvaccination was greatest in magnitude among 15 frail individuals in the sample.

\section{4- Discussion}

The main novel finding of this study is that influenza vaccination significantly reduced circulating TWEAK levels. This appears to be associated with antibody responses to specific vaccine strains and frailty.

The role of inflammation in contributing to CVDs is well documented, and substantial evidence suggests the critical involvement of TWEAK/Fn14 pathway in CVDs, both in chronic disease and at acute events [25]. Pilot data published by JAMA, 2013 [11] and those in Cochrane review [13] suggest a strong correlation between influenza vaccination and improved cardiovascular outcomes including cardiovascular morbidity and mortality. Our finding in this study suggests vaccination-related reduction of circulating TWEAK levels as a potentially important underlying molecular mechanism mediating such CVD protective effect of influenza vaccination. 
As an initial step to address whether influenza vaccine would suppress other inflammatory markers, we also measured and compared pre- and post-vaccination levels of sCD163 which is considered as an important marker primarily for monocyte/macrophage-mediated inflammation and immune activation [26]. No significant difference was observed between pre- and post-vaccination sCD163 levels, suggesting the suppressive effect of influenza vaccine on circulating TWEAK levels is rather specific. Whether influenza vaccination has broad impact on mediators of inflammation and immune activation other than sCD163 deserves further investigation.

We observed a significant association of 2-fold or higher antibody response to H1N1 vaccine strain with lower post-vaccination circulating TWEAK levels at the $\mathrm{p}<.10$ level. This observation suggests that the role of specific influenza vaccine antigens H1N1 in suppressing TWEAK-Fn14 activity warrants further investigation. From immunology perspective, antigen stimulation or load can be considered as "good" (such as vaccination) or "bad" (such as infection or injury). While severe infection (with more antigenic load and cellular damage in most cases) is known to lead worse clinical outcomes, it is less clear if more antigens in a vaccine will lead to further and broader beneficial impact. If the later is the case, we would speculate that more specific antigens in the vaccine, such as the FDAapproved higher dose (HD) influenza vaccine, would have more potent suppressive effect on TWEAK levels than the standard dose vaccine employed in this study.

We also observed a significant $(\mathrm{p}<.10)$ interactions between frailty and declines in postvaccination circulating TWEAK levels. Frail participants had a higher pre-vaccination TWEAK levels than non-frail counterparts, where frail participants $(n=15)$ had a greater decline in TWEAK compared to non-frail and pre-frail participants (Figure 3). TWEAK/ Fn14 pathway activation has been shown to have major impact on skeletal muscle biology in health and disease. For example, high TWEAK levels decreases self-renewal of progenitor cells and inhibits differentiation of myoblasts. Upon injury and in disease conditions, TWEAK attenuates regeneration of damaged myofibers and promotes fibrosis, leading to sarcopenia and skeletal muscle weakness [27]. Skeletal muscle weakness and sarcopenia along with chronic inflammation constitute the cardinal features of late-life frailty and disability with major impact on quality of life for older Americans [28, 29]. As such, influenza vaccination through its impact on the TWEAK-Fn14 pathway activity may further help improve physical function and quality of life for frail older adults.

We acknowledge the following limitations. The sample size of this study is relatively small and, this has limited the power of the study to detect potential changes in TWEAK levels associated with specific antibodies to other influenza vaccine strains. Because of the small sample size, we considered statistical effects at an alpha level of .10 as indicating an interpretable finding. Confirming these findings with a more stringent alpha level of .05 and larger sample size is an important future step. Whether the observed reduction of circulating TWEAK level is specific to influenza vaccine is currently unknown and deserves further investigation. The results described in this manuscript are our initial findings that help generate important hypotheses for future studies. On the other hand, influenza vaccination has been recommended in all elderly individuals and having 
unvaccinated controls was not possible due to ethical concerns. While seasonal, within-individual variation is possible, the interval between pre- and postvaccination blood draws was only 3-4 weeks, making such variation very unlikely. Future studies are also recommended to evaluate persistence of this effect and potential long-term effect in improving cardiovascular outcomes. In addition, we did not measure Fn14 activity or its downstream signaling. Such measurements would require biopsy of cardiac muscle and/or vessels including coronary arteries. They are, however, critically important to further investigate the TWEAK/Fn14 pathway activation. Despite such limitations, these novel data do support our original hypothesis. Reduction of circulating TWEAK levels after influenza vaccination suggests further immune modulating effect of influenza vaccine beyond influenza prevention. It is warranted to further investigate suppression of the TWEAK-Fn14 pathway as a likely important underlying biological mechanism mediating potential beneficial effect of influenza vaccination on CVDs, a major chronic condition in older adults.

\section{Acknowledgments}

Funding

This work was supported in part by NIH grant R01IA108907 (PI: Sean X. Leng] and funding from the Milstein Medical Asian American Partnership (MMAAP) Foundation (http://www.mmaapf.org) to Drs. Sean X. Leng and Min Ouyang. Dr. Min Ouyang is an Irma and Paul Milstein Program for Senior Health fellow supported by the MMAAP Foundation (http://www.mmaapf.org).

\section{Abbreviations}

TIV trivalent inactivated influenza vaccine

HA hemagglutinin

CVDs Cardiovascular Diseases

HI hemagglutination inhibition

TWEAK Tumor necrosis factor (TNF)-like weak inducer of apoptosis

HD high dose

SD standard dose

Fn14 fibroblast growth factor-inducible 14

sCD163 soluble CD163

MMSE Mini-Mental State Examination

\section{References}

1. Centers for Disease Control and Prevention (CDC). [accessed Jan 21, 2016] Heart Disease Facts on Heart Disease Statistics and Maps. Data are from the Multiple Cause of Death Files, 1999-2013, as compiled from data provided by the 57 vital statistics jurisdictions through the Vital Statistics 
Cooperative Program. <https://www.cdc.gov/dhdsp/data_statistics/fact_sheets/docs/

fs_heart_disease.pdf $>$

2. Centers for Disease Control and Prevention. [accessed Jan 21, 2016] Epidemiology and prevention of vaccine-preventable diseases, The Pink Book: Course Textbook-13th Edition. 2015. <https:// www.cdc.gov/vaccines/pubs/pinkbook/index.html>

3. Naghavi M, Barlas Z, Siadaty S, et al. Association of influenza vaccination and reduced risk of recurrent myocardial infarction. Circulation. 2000; 102(25):3039-3045. [PubMed: 11120692]

4. Siscovick DS, Raghunathan TE, Lin D, et al. Influenza vaccination and the risk of primary cardiac arrest. Am J Epidemiol. 2000; 152(7):674-7. [PubMed: 11032163]

5. Nichol KL, Nordin J, Mullooly J, et al. Influenza vaccination and reduction in hospitalizations for cardiac disease and stroke among the elderly. N Engl J Med. 2003; 348(14):1322-32. [PubMed: 12672859]

6. Smeeth L, Thomas SL, Hall AJ, et al. Risk of myocardial infarction and stroke after acute infection or vaccination. N Engl J Med. 2004; 351(25):2611-8. [PubMed: 15602021]

7. Keshtkar-Jahromi M, Vakili H, Rahnavardi M, et al. The efficacy of influenza vaccination in reducing cardiovascular events in patients with coronary artery diseases: IVCAD study. Clin Microbiol Infect. 2009; 15:395. [PubMed: 19489920]

8. Hung IF, Leung AY, Chu DW, et al. Prevention of acute myocardial infarction and stroke among elderly persons by dual pneumococcal and influenza vaccination: a prospective cohort study. Clin Infect Dis. 2010; 51(9):1007-1016. [PubMed: 20887208]

9. Siriwardena AN, Gwini SM, Coupland CA. Influenza vaccination, pneumococcal vaccination, and risk of acute myocardial infarction: matched case-control study. CMAJ. 2010; 182(15):1617-23. [PubMed: 20855479]

10. Macintyre CR, Heywood AE, Kovoor P, et al. Ischaemic heart disease, influenza and influenza vaccination: a prospective case control study. Heart. 2013; 99(24):1843-8. [PubMed: 23966030]

11. Udell JA, Zawi R, Bhatt DL, et al. Association between influenza vaccination and cardiovascular outcomes in high-risk patients: a meta-analysis. JAMA. 2013; 310(16):1711-20. [PubMed: 24150467]

12. Loomba RS, Aggarwal S, Shah PH, et al. Influenza vaccination and cardiovascular morbidity and mortality: analysis of 292,383 patients. J Cardiovasc Pharmacol Ther. 2012; 17(3):277-83. [PubMed: 22172681]

13. Clar C, Oseni Z, Flowers N, et al. Influenza vaccines for preventing cardiovascular disease. Cochrane Database Syst Rev. 2015; 5

14. Chicheportiche Y, Bourdon PR, Xu H, et al. TWEAK, a new secreted ligand in the tumor necrosis factor family that weakly induces apoptosis. J Biol Chem. 1997; 272(51):32401-10. [PubMed: 9405449]

15. Wiley SR, Cassiano L, Lofton T, et al. A novel TNF receptor family member binds TWEAK and is implicated in angiogenesis. Immunity. 2001; 15(5):837-46. [PubMed: 11728344]

16. Winkles JA. The TWEAK-Fn14 cytokine receptor axis: discovery, biology and therapeutic targeting. Nat Rev Drug Discov. 2008; 7(5):411-25. [PubMed: 18404150]

17. Kim SH, Kang YJ, Kim WJ, et al. TWEAK can induce proinflammatory cytokines and matrix metalloproteinase-9 in macrophages. Circ J. 2004; 68(4):396-9. [PubMed: 15056843]

18. Chorianopoulos E, Jarr K, Steen H, et al. Soluble TWEAK is markedly upregulated in patients with ST-elevation myocardial infarction and related to an adverse short-term outcome. Atherosclerosis. 2010; 211(1):322-6. [PubMed: 20303491]

19. Novoyatleva T, Wiebke J, Wietelmann A, et al. TWEAK/Fn14 axis is a positive regulator of cardiac hypertrophy. Cytokine. 2013; 64:43-45. [PubMed: 23764551]

20. Sastre C, Fernández-Laso V, Madrigal-Matute J, et al. Genetic deletion or TWEAK blocking antibody administration reduce atherosclerosis and enhance plaque stability in mice. J Cell Mol Med. 2014; 18(4):721-34. [PubMed: 24479820]

21. Blanco-Colio LM. TWEAK/Fn14 Axis: A Promising Target for the Treatment of Cardiovascular Diseases. Front Immunol. 2014; 5(3) 
22. Yao X, Hamilton RG, Weng NP, et al. Frailty is associated with impairment of vaccine-induced antibody response and increase in post-vaccination influenza infection in community-dwelling older adults. Vaccine. 2011; 29(31):5015-21. [PubMed: 21565245]

23. Fried LP, Tangen CM, Walston J, et al. Frailty in older adults: evidence for a phenotype. J Gerontol A Biol Sci Med Sci. 2001; 56(3):M146-56. [PubMed: 11253156]

24. Pangman VC, Sloan J, Guse L. An examination of psychometric properties of the mini-mental state examination and the standardized mini-mental state examination: implications for clinical practice. Appl Nurs Res. 2000; 13(4):209-13. [PubMed: 11078787]

25. Novoyatleva T, Sajjad A, Engel FB. TWEAK-Fn14 Cytokine-Receptor Axis: A New Player of Myocardial Remodeling and Cardiac Failure. Front Immunol. 2014; 5:50. [PubMed: 24611063]

26. Moller HJ, Aerts H, Grønbaek H, et al. Soluble CD163: a marker molecule for monocyte/ macrophage activity in disease. Scand J Clin Lab Invest Suppl. 2002; 237:29-33. [PubMed: 12570164]

27. Tajrishi MM, Zheng TS, Burkly LC, et al. The TWEAK-Fn14 pathway: a potent regulator of skeletal muscle biology in health and disease. Cytokine Growth Factor Rev. 2014; 25(2):215-25. [PubMed: 24444596]

28. Leng SX, Xue Q, Tian J, et al. Inflammation and frailty in older women. J Am Geriatr Soc. 2007; 55:864-71. [PubMed: 17537086]

29. Chen XJ, Mao GX, Leng SX. Frailty syndrome: an overview. Clin Interv Aging. 2014; 9:433-41. [PubMed: 24672230] 


\section{Highlights}

- $\quad$ This pilot study showed that influenza vaccine reduces TWEAK levels in older adults.

- This appears to be associated with antibody responses to vaccine strains and frailty.

- These novel findings suggest suppression of TWEAK/Fn14 pathway as a potential mechanism.

- This could mediate beneficial effects of influenza vaccine against cardiovascular disease. 
Screening (Early October 2007, N=94)

- Older than 70

- Frailty Screening

- Community dwelling

-Exculsion criteria

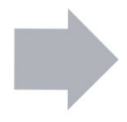

Visit 1 (Early to mid

November 2007, $\mathrm{N}=78$ )

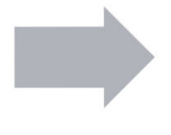

Visit 2 (Early to mid

November 2007, $\mathrm{N}=69$ )

-4th week after vaccination

- History

-Blood collection

Fig. 1.

Schematic illustration of the study protocol including pre-vaccination screening, Influenza immunization (Visit 1), and Post-vaccination blood draw (Visit 2). 


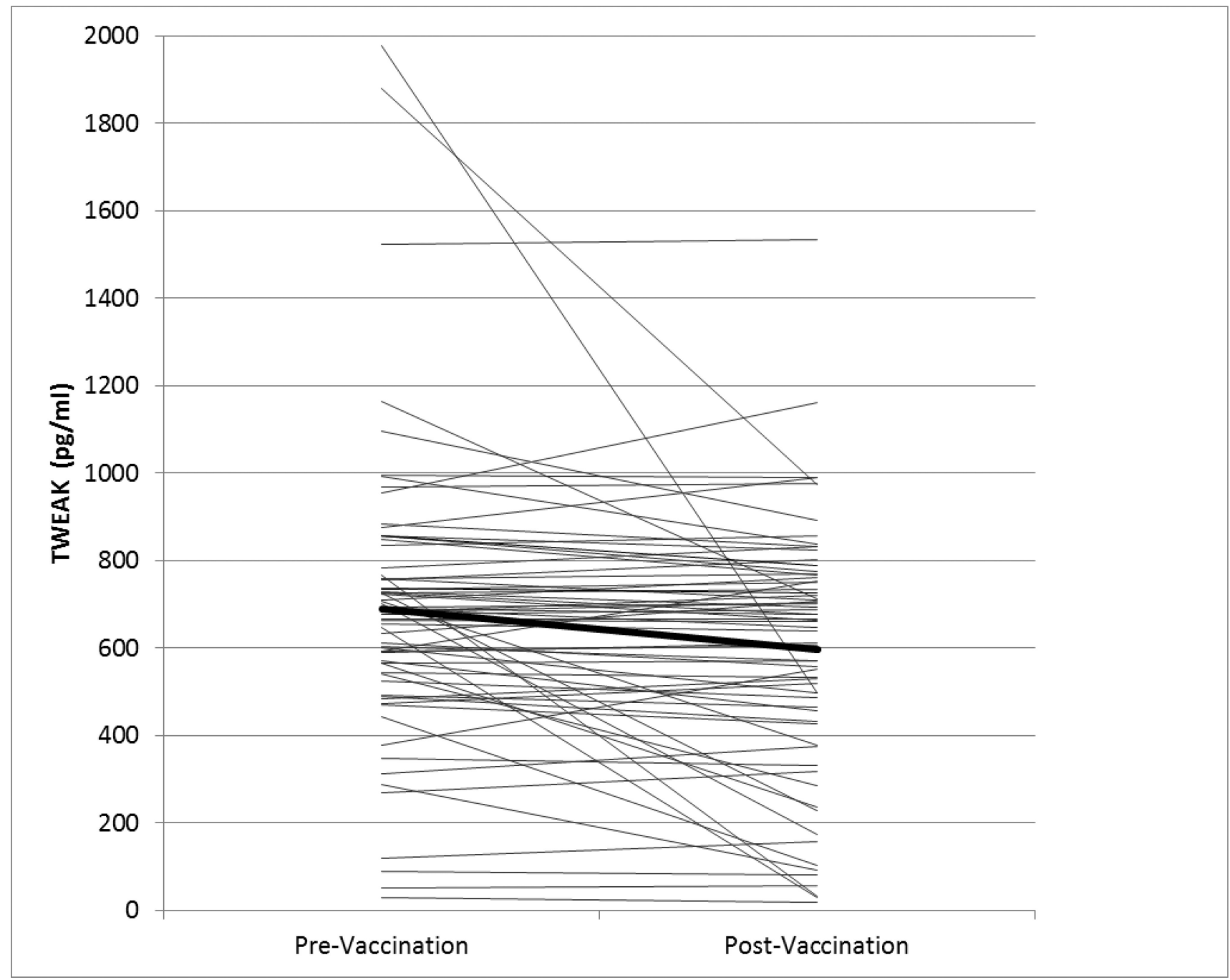

Fig. 2.

TWEAK levels were measured in pre- and post-vaccination serum samples collected from the same individuals. Levels are shown for each patient. The difference between pre- and post-vaccination levels is statistically significant $(\mathrm{p}=.003)$. 


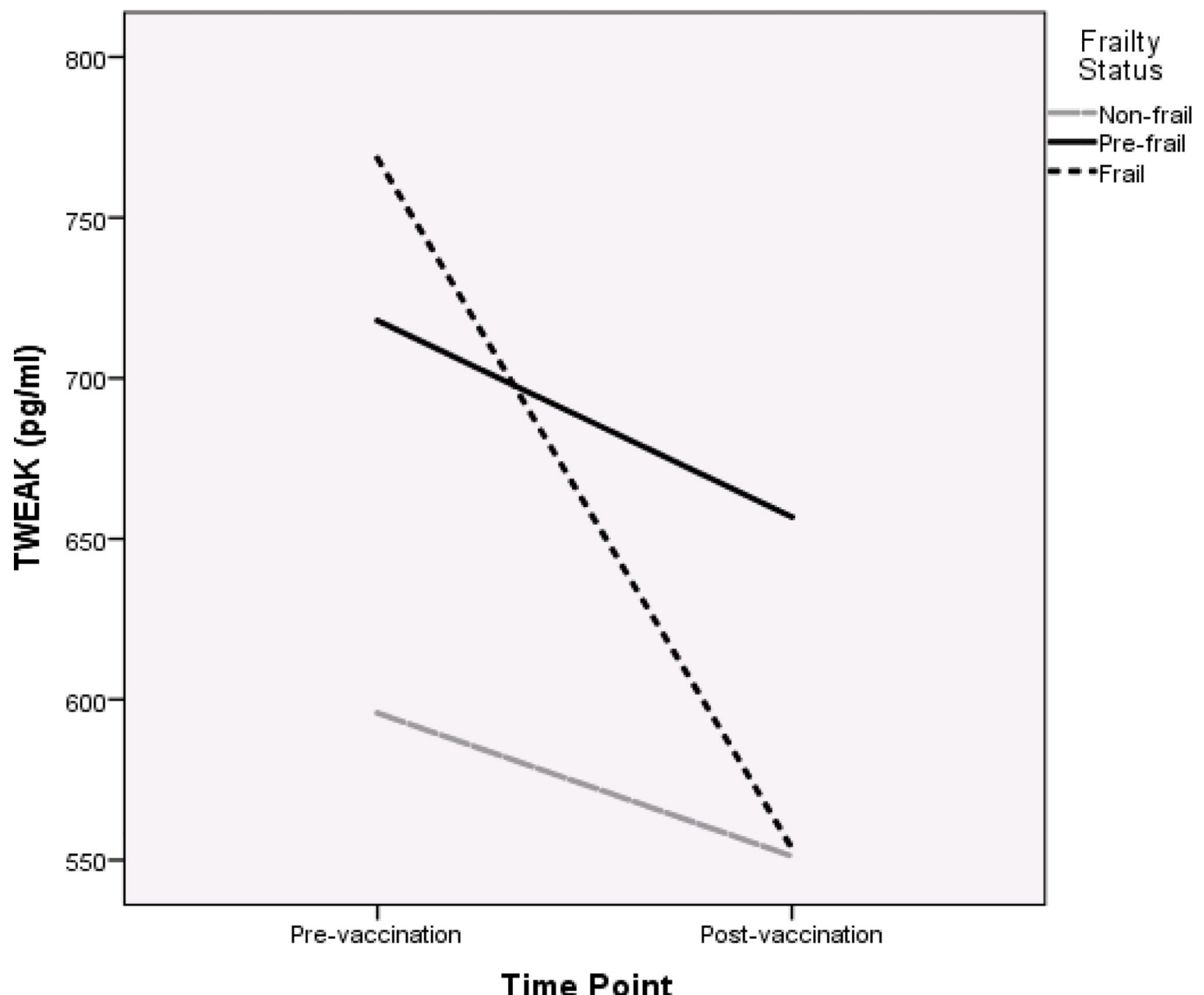

Fig. 3.

Pre and Post-vaccination TWEAK levels (pg/ml) by Frailty status. The vaccination-induced reduction of TWEAK levels was greatest among frail individuals. 
Table 1

Selected demographic and clinical characteristics of the study participants ( $n=69)$

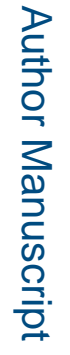

\begin{tabular}{|c|c|}
\hline Variable & $\mathbf{N}(\%)$ \\
\hline \multicolumn{2}{|l|}{ Race } \\
\hline White & $63(91 \%)$ \\
\hline Other & $6(9 \%)$ \\
\hline Sex, Females & $54(78 \%)$ \\
\hline Age (years), mean $( \pm \mathrm{SD})$ & $84.6( \pm 4.6)$ \\
\hline \multicolumn{2}{|l|}{ Education } \\
\hline High school or less & $13(19 \%)$ \\
\hline Some college & $13(19 \%)$ \\
\hline College or some graduate work & $23(33 \%)$ \\
\hline Post-graduate school & $20(29 \%)$ \\
\hline \multicolumn{2}{|l|}{ Frailty status ${ }^{*}$} \\
\hline Non-frail & $22(32 \%)$ \\
\hline Pre-frail & $31(46 \%)$ \\
\hline Frail & $15(22 \%)$ \\
\hline \multicolumn{2}{|l|}{ MMSE Score ${ }^{*}$} \\
\hline 24 and up & $66(97 \%)$ \\
\hline Less than 24 & $2(3 \%)$ \\
\hline \multicolumn{2}{|l|}{ Common Clinical Conditions } \\
\hline Angina & $5(7 \%)$ \\
\hline Cancer & $21(30 \%)$ \\
\hline Congestive Heart Failure & $3(4 \%)$ \\
\hline History of Heart Attack & $14(20 \%)$ \\
\hline Hypertension * & $23(33 \%)$ \\
\hline History of Stroke & $14(20 \%)$ \\
\hline Diabetes Mellitus & $8(12 \%)$ \\
\hline
\end{tabular}

Notes.

* One missing value on Frailty status and MMSE Score. 


\section{Table 2}

Multiple regression of post-vaccination TWEAK levels on antibody responses to influenza vaccine H1N1 strain $^{*}$

\begin{tabular}{|l|l|l|l|}
\hline Variable & Unstandardized beta (SE) & 95 CI & p value \\
\hline Intercept & $729.4(85.1)$ & $559.2,899.6$ & $<.001$ \\
\hline Antibody responses to H1N1 (1-fold vs. 2-fold or greater) & $-92.1(53.6)$ & $-199.3,15.2$ & .091 \\
\hline TWEAK levels (pg/ml), pre-vaccine & $0.51(.08)$ & $0.35,0.67$ & $<.001$ \\
\hline Sex & $-162.2(63.3)$ & $-288.8,-35.5$ & .013 \\
\hline Age (years) & $-1.63(5.99)$ & $-13.6,13.4$ & .79 \\
\hline Hypertension & $-62.6(57.2)$ & $-176.9,51.7$ & .28 \\
\hline
\end{tabular}

* Adjusted for age, sex, hypertension, and frailty status 